\title{
Sirtuin 3-dependent mitochondrial dynamic improvements protect against acute kidney injury
}

\author{
Marina Morigi, ${ }^{1}$ Luca Perico, ${ }^{1}$ Cinzia Rota, ${ }^{1}$ Lorena Longaretti, ${ }^{1}$ Sara Conti, ${ }^{1}$ Daniela Rottoli, ${ }^{1}$ Rubina Novelli, ${ }^{1}$ \\ Giuseppe Remuzzi, ${ }^{1,2}$ and Ariela Benigni ${ }^{1}$ \\ ${ }^{1}$ IRCCS - Istituto di Ricerche Farmacologiche "Mario Negri," Centro Anna Maria Astori, Science \& Technology Park Kilometro Rosso, Bergamo, Italy. unit of Nephrology and Dialysis, \\ Azienda Ospedaliera Papa Giovanni XXIII, Bergamo, Italy.
}

\begin{abstract}
Acute kidney injury (AKI) is a public health concern with an annual mortality rate that exceeds those of breast and prostate cancer, heart failure, and diabetes combined. Oxidative stress and mitochondrial damage are drivers of AKI-associated pathology; however, the pathways that mediate these events are poorly defined. Here, using a murine cisplatin-induced AKI model, we determined that both oxidative stress and mitochondrial damage are associated with reduced levels of renal sirtuin 3 (SIRT3). Treatment with the AMPK agonist AICAR or the antioxidant agent acetyl-L-carnitine (ALCAR) restored SIRT3 expression and activity, improved renal function, and decreased tubular injury in WT animals, but had no effect in Sirt3 ${ }^{-/-}$ mice. Moreover, Sirt3-deficient mice given cisplatin experienced more severe AKI than WT animals and died, and neither AICAR nor ALCAR treatment prevented death in Sirt3 ${ }^{-/-}$AKI mice. In cultured human tubular cells, cisplatin reduced SIRT3, resulting in mitochondrial fragmentation, while restoration of SIRT3 with AICAR and ALCAR improved cisplatin-induced mitochondrial dysfunction. Together, our results indicate that SIRT3 is protective against AKI and suggest that enhancing SIRT3 to improve mitochondrial dynamics has potential as a strategy for improving outcomes of renal injury.
\end{abstract}

\section{Introduction}

Worldwide, 13.3 million people experience acute kidney injury (AKI) every year. AKI is associated with a high mortality rate, the development of long-term chronic kidney disease, and other types of organ dysfunction in a substantial percentage of patients. The annual mortality rate for AKI currently exceeds $50 \%$, at least in patients requiring early renal replacement therapy, and is greater than the mortality rate for breast and prostate cancer, heart failure, and diabetes together (1). Extensive research in the last 20 years and spectacular advances in pathophysiology, however, have not translated so far into effective therapeutic tools that could improve survival after an AKI episode. AKI is thus still a major public health issue, leading to thousands of unnecessary death in the United Kingdom alone, according to a recent National Institute for Health and Care Excellence (NICE) survey (2). One of the critical issues in designing innovative therapies capable of hastening renal repair is that the plausible key mediator of damage amenable for pharmacological manipulation has not been identified so far. Studies in this direction are urgently needed because the short- and long-term effects of AKI (again, according to NICE) cost the British National Health Service $£ 424-£ 620$ million, more than it spends on breast, lung, and skin cancer combined - a heavy societal burden.

After an ischemic or toxic challenge (3-5), dysfunction and the loss of tubular epithelial cells play a critical role in the evolution of AKI. In response to damage, tubular cells lose cytoskeletal integrity and cell polarity, with the mislocation of membrane proteins leading to the disruption of cell-matrix and cell-cell interaction (3-5).

Conflict of interest: The authors have declared that no conflict of interest exists. Submitted: June 19, 2014; Accepted: December 10, 2014.

Reference information: J Clin Invest. 2015;125(2):715-726. doi:10.1172/JCI77632.
Central to tubular injury is mitochondrial dysregulation $(3,6)$, manifested in a reduction in cell respiration and ATP production (4). Mitochondrial function rests on a complex molecular machinery finely tuned and balanced by regulatory proteins of the two opposing processes fission and fusion (6-9). In experimental AKI, fission predominates, resulting in mitochondrial fragmentation, outer membrane permeabilization, and the release of apoptogenic factors $(6,9)$, which together with ROS overproduction (6) determine tubular epithelial cell injury and apoptosis. Novel strategies for preventing and treating AKI may therefore derive from studies targeting regulators of mitochondrial dynamics. In this context we thought that looking at sirtuins (SIRTs) - which regulate energy metabolism and oxidative stress (10) - could be of great interest.

Sirtuins are an evolutionarily conserved family comprising 7 proteins with $\mathrm{NAD}^{+}$-dependent deacetylase activity in mammals, 3 of which (SIRT3-SIRT5) are mainly localized in the mitochondrion. Robust mitochondrial protein hyperacetylation in Sirt3 ${ }^{-1-}$ mice points to SIRT3 as the major mitochondrial deacetylase (11) that maintains basal ATP levels by direct physical association with complex I (12) and the activation of $\mathrm{F}_{0} \mathrm{~F}_{1}$ ATPase (13), as well as ROS homeostasis through the regulation of detoxifying enzymes $(14,15)$. SIRT3 overexpression protects cells against BAX-mediated apoptosis, acting on NF- $\mathrm{kB}$ downstream target genes such as manganese SOD (MnSOD) and BCL2, thus making cells resistant to hydrogen peroxide-mediated cell injury (16). In previous studies we found that elevated renal expression of SIRT3 reduces ROS and ameliorates mitochondria dynamics that translate into the longevity phenotype in mice deficient for angiotensin II type 1 receptor (17). The question of whether renal SIRT3 could be a master regulator of injury and repair in AKI represented a logical extension of the above studies. 

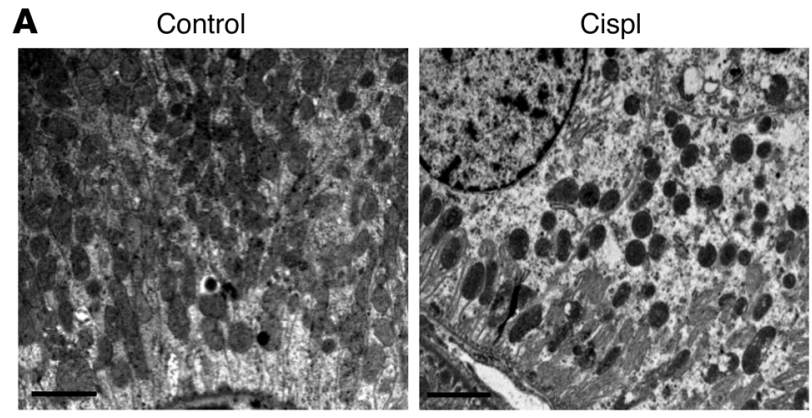

D

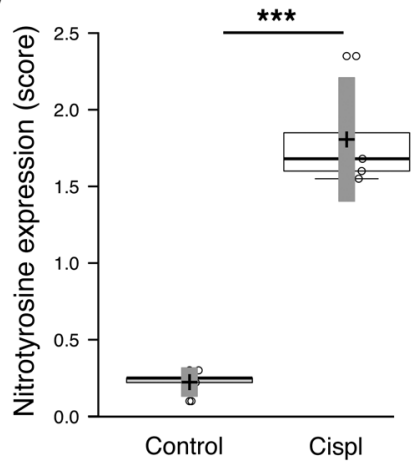

$\mathbf{E}$

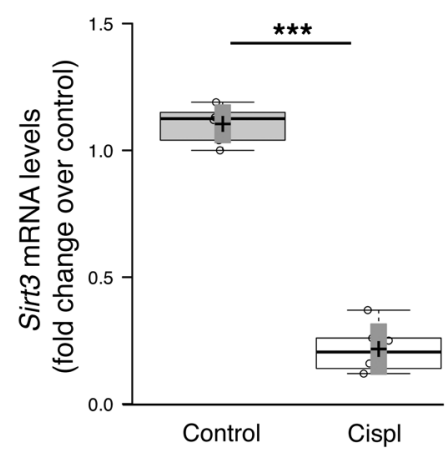

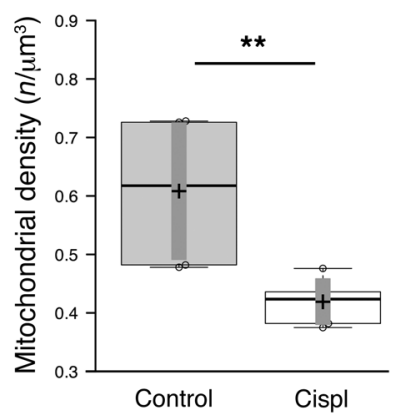

C

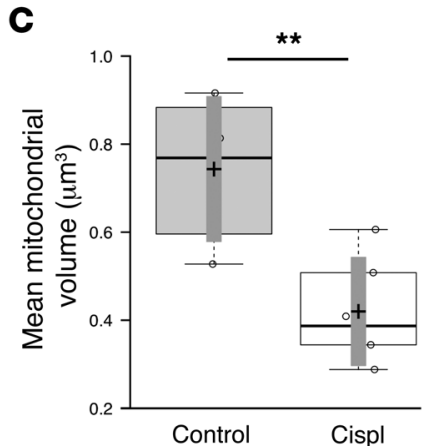

Figure 1. Mitochondrial dysregulation and SIRT3 expression in mice with cisplatin-induced AKI. (A) Representative transmission electron micrographs of the ultrastructure of mouse proximal tubular cells obtained from resin-embedded kidney sections of control and cisplatin-treated (Cispl) mice at 4 days. Scale bars: $2 \mu \mathrm{m}$. (B and C) Quantification of mitochondria per volume $\left(n / \mu \mathrm{m}^{3}\right)$ and mean mitochondrial volume $\left(\mu \mathrm{m}^{3}\right)$ by morphometric analysis in proximal tubular cells of control and cisplatin-treated mice. ${ }^{*} P<0.01$, unpaired Student's $t$ test. $n=6$ mice per group. (D) Staining score of nitrotyrosine, a marker of peroxynitrite, assessed in kidney cortex of control and cisplatin-treated mice. ${ }^{* * *} P<0.001$, unpaired Student's $t$ test. $n=5$ mice per group. (E) Whole kidney expression of Sirt3 mRNA analyzed by real-time PCR in control and cisplatin-treated mice. ${ }^{* * *} P<0.001$, unpaired Student's $t$ test. $n=6$ mice per group.

Here we report that in mice with cisplatin-induced AKI, tubular cell mitochondrial abnormalities were associated with decreased renal SIRT3 mRNA and protein expression. Mice with cisplatin-induced AKI treated with 5-aminoimidazole-4-carboxamide-1- $\beta$-D-ribofuranoside (AICAR), an activator of AMPK, or the antioxidant agent acetyl-L-carnitine (ALCAR) experienced improved renal function and less tubular injury via the restoration of mitochondrial SIRT3 expression and activity. Sirt3-deficient mice given cisplatin had more severe AKI and died, in contrast to WT animals. Moreover, Sirt3 ${ }^{-/}$AKI mice were not protected from kidney injury and death by either AICAR or ALCAR. In vitro data obtained from cisplatin-damaged proximal tubular cells clearly indicate a functional role for SIRT3 in preserving mitochondrial integrity by preventing dynamin-related protein-dependent (DRP1-dependent) fission, loss of membrane potential, and PTEN-induced putative kinase 1-related (PINK1-related) mitophagy. These selective rescue mechanisms point the way toward a new therapeutic target for the treatment of AKI.

\section{Results}

SIRT3 is reduced in cisplatin-induced AKI. Four days after cisplatin exposure, mice developed AKI, characterized by proximal tubular injury (18). Electron micrographs of proximal tubules showed mitochondrial fragmentation into short rods or spheres and rarefaction compared with the elongated organelles observed in the control tissue (Figure 1A). Quantification through morphometric analysis revealed that both the number and volume of mitochondria were reduced in AKI mice compared with controls (Figure 1, B and C). Since ROS cause mitochondria damage and dysfunctional signaling resulting in changes in mitochondrial dynamics (6), we evaluated the expression of nitrotyrosine, a marker of peroxynitrite. An 8 -fold increase in nitrotyrosine staining was observed in proximal tubules of mice with cisplatin-induced AKI compared with control mice (Figure 1D). Parallel to this, the mRNA and protein expression of SIRT3 was reduced by $80 \%$ in kidneys of cisplatin-treated mice compared with control animals (Figure 1E and Figure 2E). Concomitant with decreased SIRT3 levels, a significant reduction in the transcript levels of nicotinamide phosphoribosyltransferase (Nampt) $(0.34 \pm 0.1$-fold change in cisplatin-treated mice relative to control $[1 \pm 0.2], P<0.01$, the rate-limiting enzyme in the biosynthesis of the SIRT3 substrate $\mathrm{NAD}^{+}(19)$, was found. In addition, the expression of the transcription factor peroxisome proliferator-activated receptor $\gamma$ coactivator-1 $\alpha(\operatorname{Pgcla})$, a key inducer of ROS-detoxifying enzymes (15) and mitochondrial biogenesis (14), was reduced in cisplatin-treated mice $(0.21 \pm 0.1$-fold change in cisplatin-treated mice relative to control $[1 \pm 0.1], P<0.01)$.

Increasing SIRT3 using AMPK agonist protects from cisplatininduced AKI. AMPK is an upstream signal of SIRT3 that regulates gene expression and the activity of $\operatorname{NAMPT}(20,21)$ and PGC-1 $\alpha$ $(22,23)$, and due to the lack of availability of specific SIRT3 activators, we evaluated whether the AMPK activator AICAR protected animals from cisplatin-induced AKI via the induction of SIRT3. 
A

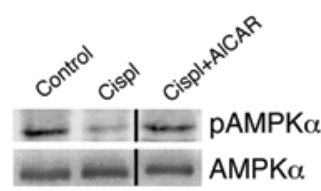

B

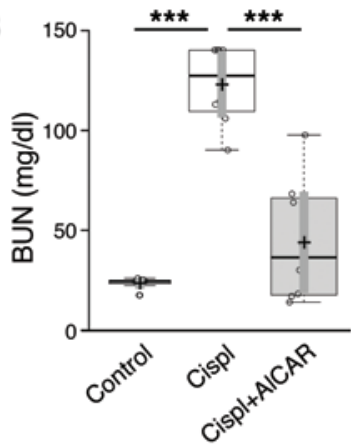

C
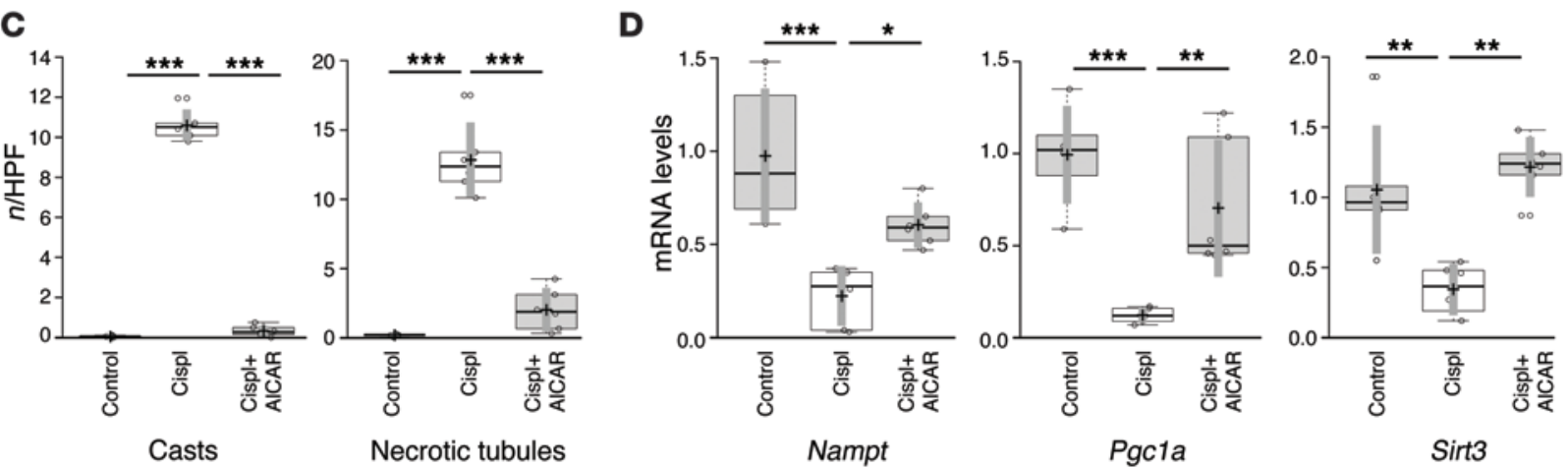

$\mathbf{E}$

Control
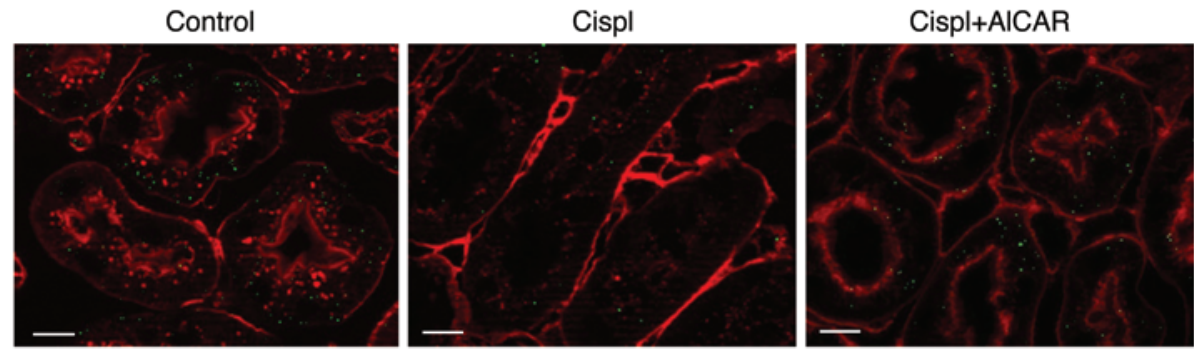

Figure 2. AMPK agonist AICAR exerts renoprotection in mice with AKI by increasing SIRT3. (A) Western blot and densitometric analysis of pAMPK $\alpha$ and total AMPK $\alpha$ in the renal tissue of control and cisplatin-treated mice given saline or AICAR at 4 days. ${ }^{*} P<0.05$, ANOVA corrected with Bonferroni coefficient. $n=5$ control mice; $n=7$ cisplatin mice+saline; $n=6$ cisplatin mice+AICAR. (B) Renal function in control mice and cisplatin-treated mice receiving saline or AICAR at 4 days. ${ }^{* * *} P<0.001$, ANOVA corrected with Bonferroni coefficient. $n=7$ control mice; $n=8$ cisplatin mice+saline; $n=8$ cisplatin mice+AICAR. (C) Effect of AICAR treatment on renal histological changes at 4 days evaluated as number of cast and necrotic tubules per HPF. ${ }^{* * *} P<0.001$, ANOVA corrected with Bonferroni coefficient. $n=6$ mice per group. (D) Renal expression of Nampt, Pgc1a, and Sirt3 transcripts studied by real-time PCR in control and cisplatin-treated mice given saline or AICAR at 4 days. ${ }^{*} P<0.05$, ${ }^{* *} P<0.01$, and ${ }^{* * *} P<0.001$, ANOVA corrected with Bonferroni coefficient. $n=6$ mice per group. (E) Representative images of SIRT3 protein staining (green) in proximal tubules of control and cisplatin-treated mice given saline or AICAR at 4 days. Scale bars: $10 \mu \mathrm{m}$.

AMPK activity, documented as the ratio of phosphorylated AMPKo (pAMPK $\alpha$ ) to AMPK $\alpha$, decreased by $61 \%$ in renal tissue of cisplatin-treated mice, compared with control mice, and was restored by AICAR (Figure 2A). In AKI mice, the AMPK activator AICAR also restored renal function, evaluated as blood urea nitrogen (BUN) and serum creatinine, to levels comparable to those in controls (Figure 2B and Supplemental Table 1; supplemental material available online with this article; doi:10.1172/JCI77632DS1) and ameliorated tubular injury in terms of reduction of hyaline casts and tubular cell degeneration and necrosis (Figure 2C and Supplemental Figure 1). The protective effect of AICAR was associated with a robust upregulation of transcript levels of Nampt, Pgcla, and Sirt3 in renal tissue (Figure 2D). Consistent with the gene expression data, staining of SIRT3 protein in injured proximal tubular cells was reduced compared with controls (SIRT3-positive area, cisplatin: $10.54 \pm 0.5$ vs. control: $30.7 \pm 0.4 \mu \mathrm{m}^{2} /$ high-power field [HPF], $P<0.001$ ) and was significantly recovered by AICAR treatment $\left(18.7 \pm 0.4 \mu \mathrm{m}^{2} / \mathrm{HPF}, P<0.001 \mathrm{vs}\right.$. cisplatin+saline and control) (Figure 2E). Notably, costaining SIRT3 with the voltagedependent anion channel (VDAC) mitochondrial marker showed a robust and specific finding of SIRT3 localization in the organelles (Supplemental Figure 2).

Immunogold labeling of SIRT3 allowed for the identification of a discrete amount of protein exclusively localized within proximal tubular cell mitochondria in control animals (Figure 3A). Gold particles were either absent in AKI mice given saline or barely detectable in a few scattered mitochondria. AICAR treatment fully reconstituted the SIRT3 mitochondrial expression pattern (Figure 3A). Consistent with this finding, AICAR treatment also protected from mitochondrial fragmentation by normalizing mitochondrial 

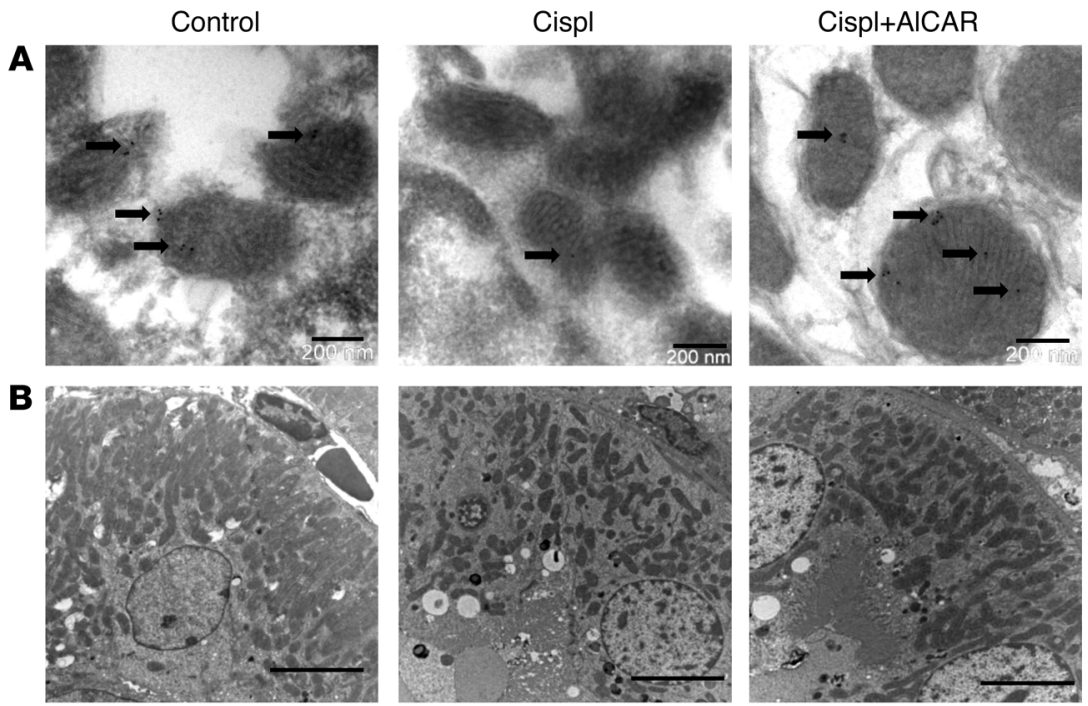

C
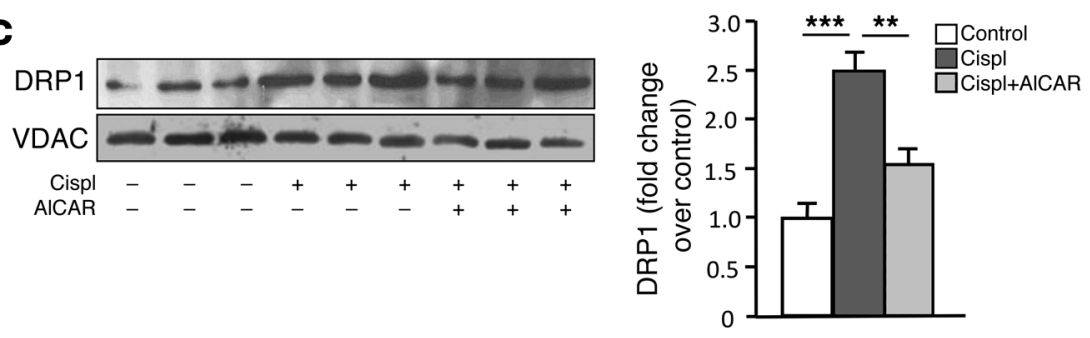

D

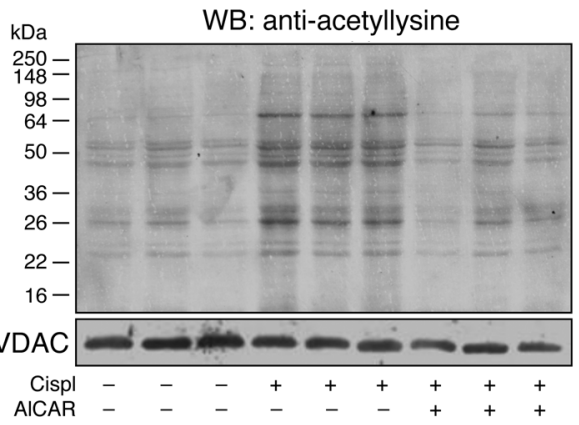

Figure 3. AICAR limits mitochondrial fragmentation and restores SIRT3 protein and activity. (A) Pattern of immunogold labeling for SIRT3 (arrows) in tubular cell mitochondria of control and cisplatin-treated mice given saline or AICAR at 4 days. Scale bars: $200 \mathrm{~nm}$. (B) Representative transmission electron micrographs of the proximal tubular cell ultrastructure obtained from kidney sections of control and cisplatin-treated mice given saline or AICAR at 4 days. Scale bars: 5,000 nm. (C) Western blot and densitometric analysis of DRP1 protein expression in purified mitochondrial fraction isolated from renal tissue of control and cisplatin-treated mice given saline or AICAR at 4 days. VDAC protein expression was used as a sample loading control on the same membrane after stripping. ${ }^{* *} P<0.01$ and ${ }^{* *} P<0.001$, ANOVA corrected with Bonferroni coefficient. Values are mean \pm SEM $(n=3$ mice per group). (D) Western blot (WB) and densitometric analysis of protein acetylation in mitochondria isolated from renal tissue of control and cisplatin-treated mice given saline or AICAR at 4 days. VDAC protein expression was used as a sample loading control on the same membrane after stripping. ${ }^{*} P<0.05$ and ${ }^{* *} P<0.01$, ANOVA corrected with Bonferroni coefficient. Values are mean \pm SEM ( $n=3$ mice per group). VDAC blots reported in $\mathbf{C}$ and D derived from the same gel. density (Supplemental Table 2), as observed at the ultrastructural level in AKI mice (Figure 3B).

To unravel whether the mitochondrial fragmentation observed in cisplatin-damaged tubules could be the result of activated fission, we studied DRP1, which translocates to mitochondria early during acute tubular cell injury (9). As shown in Figure 3C, a marked accumulation of the DRP1 protein was documented in the mitochondrial fraction from renal tissue of cisplatin-treated mice compared with control mice (2.5-fold increase). Treatment with AICAR reduced mitochondrial DRP1 protein translocation (Figure 3C).

To investigate whether AICAR modulated the deacetylase activity of SIRT3, we purified mitochondria from the renal tissues of control and cisplatin-exposed mice treated with saline or AICAR. Using a specific antibody that detects protein acetylation at lysine residues, we found that acetylation of several proteins with molecular weights of approximately $64,55,52,48,34,28$, 26 , and $22 \mathrm{kDa}$ (Figure 3D) was 9-fold higher in renal mitochondria of cisplatin-treated than control mice (Figure 3D). Treatment with AICAR normalized the acetylation of mitochondrial proteins (Figure 3D), indicating that this AMPK agonist restores SIRT3 deacetylase activity.

Increasing SIRT3 using the antioxidant ALCAR protects from cisplatin-induced AKI. The close relationship between oxidative damage and mitochondrial fragmentation in AKI (6) prompted us to investigate the effects of ALCAR (known to modulate mitochondrial antioxidant activity, apoptosis, and inflammation; ref. 24) on SIRT3 signaling in cisplatin-treated mice with AKI. Similar to the effects observed with the AMPK agonist, ALCAR markedly improved renal function in AKI mice compared with mice given saline (Figure 4A and Supplemental Table 1). Renal protection was accompanied by the modulation of intrarenal expression of the transcripts for Nampt, Pgcla, and Sirt3, which were significantly increased in cisplatin-exposed mice after ALCAR treatment (Figure $4 \mathrm{~B})$. Changes in gene expression were paralleled by increased SIRT3 protein expression in response to ALCAR treatment (Supplemental Figure 3). ALCAR attenuated severe tubular cell damage in the kidney of mice with AKI (Supplemental Figure 1). At the ultrastructural level, the protective effect of ALCAR resulted in 

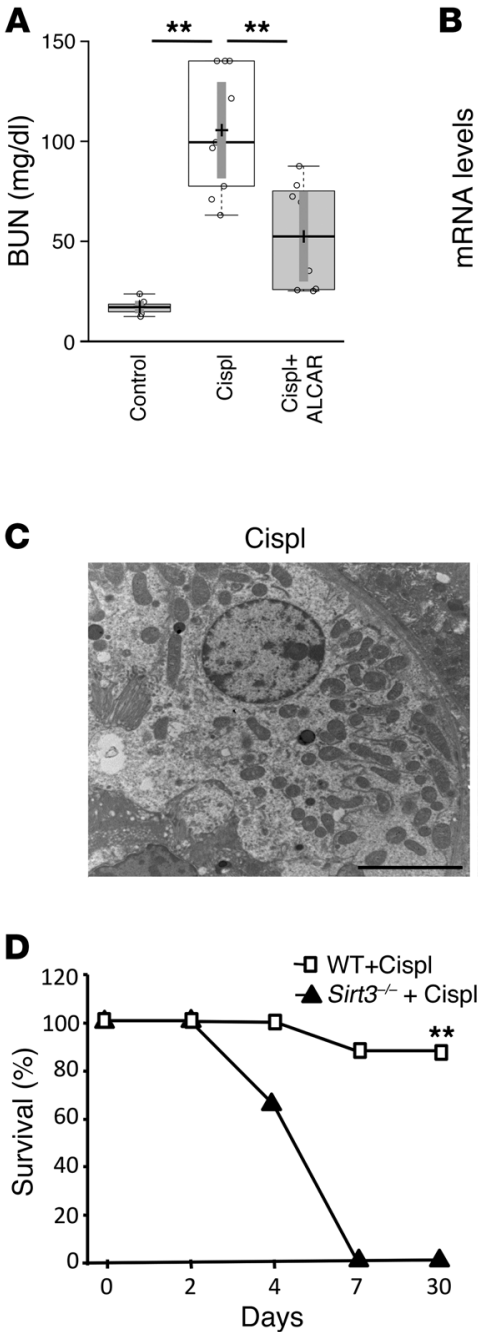
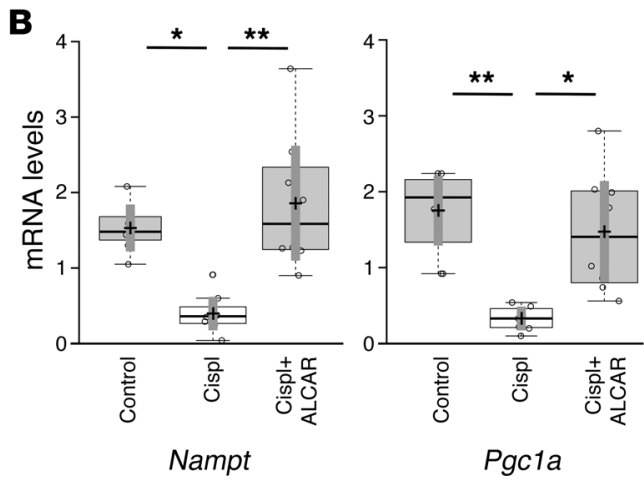

Pgc1a

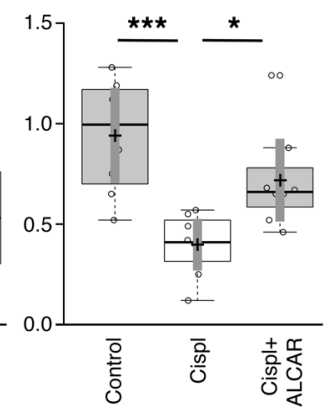

Sirt3
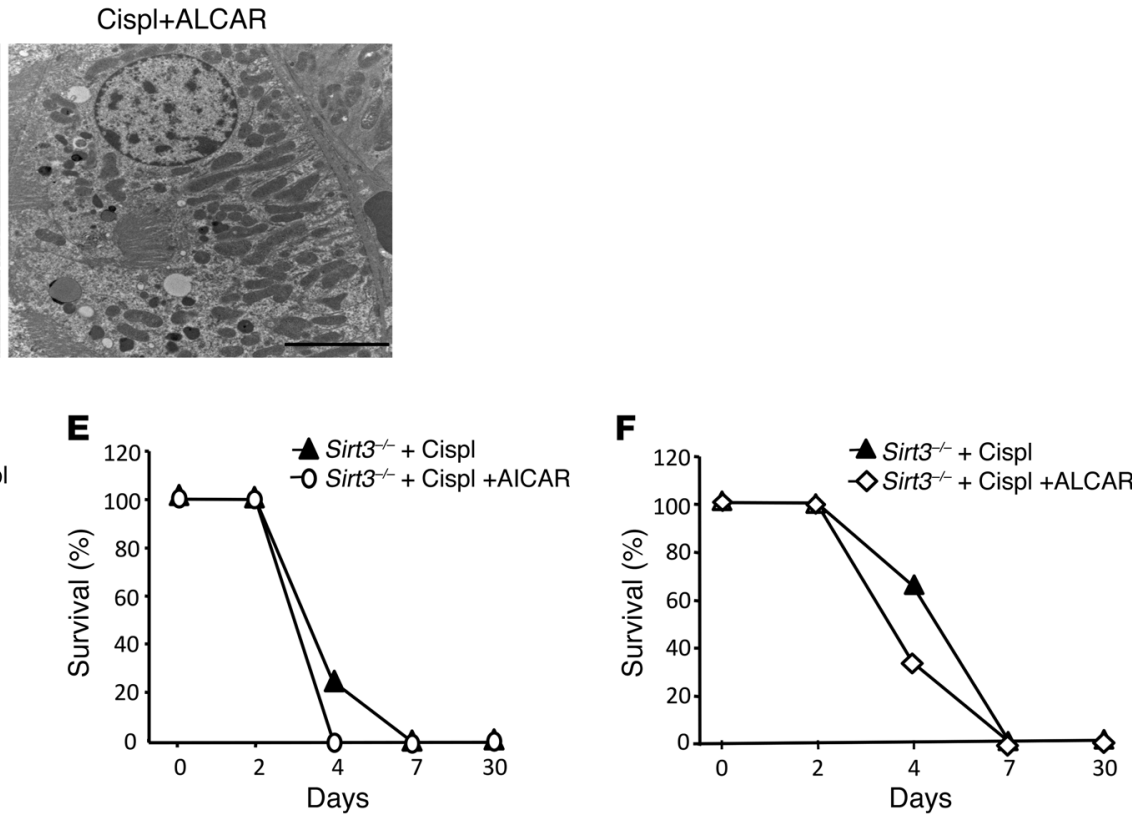

Figure 4. The antioxidant ALCAR protects mice from AKI by enhancing SIRT3. (A) Renal function in control and cisplatin-treated mice given saline or ALCAR at 4 days. ${ }^{* *} P<0.01$, ANOVA corrected with Bonferroni coefficient. $n=8$ control mice; $n=8$ cisplatin mice + saline; $n=9$ cisplatin mice + ALCAR. (B) Whole kidney expression of Nampt, Pgc1a, and Sirt3 mRNA analyzed by real-time PCR in control and cisplatin-treated mice given saline or ALCAR at 4 days. ${ }^{*} P<0.05,{ }^{* *} P<0.01$, and ${ }^{* * *} P<0.001$, ANOVA corrected with Bonferroni coefficient. $n=7-8$ mice for each group. (C) Representative transmission electron micrographs of the proximal tubular cell ultrastructure from kidney sections of cisplatin-treated mice given saline or ALCAR at 4 days. Scale bars: $5,000 \mathrm{~nm}$. (D) Survival curves of WT and Sirt3 ${ }^{-/-}$mice with cisplatin-induced AKI. ${ }^{* *} P<0.01$, log-rank test. $n=8$ WT cisplatin-treated mice; $n=7$

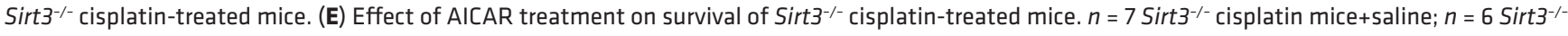
cisplatin mice+AICAR. (F) Effect of ALCAR treatment on survival of Sirt3 ${ }^{-1-}$ cisplatin-treated mice. $n=8$ Sirt3 ${ }^{-/-}$cisplatin mice + saline; $n=6$ Sirt3 ${ }^{-1-}$ cisplatin mice+ALCAR. Values are mean \pm SEM.

a reduction in the mitochondrial fragmentation observed in AKI mice (Figure $4 \mathrm{C}$ and Supplemental Table 2).

SIRT3 deficiency exacerbates AKI. The biological and clinical relevance of SIRT3 in AKI was further documented in Sirt3 $3^{-1-}$ mice. In these animals, cisplatin induced earlier and remarkably more severe renal dysfunction compared with that in WT animals (Supplemental Figure 4). Moreover, the kidney tubular injury score at 4 days revealed that Sirt $3^{--}$were more severely affected than WT mice (necrotic tubules/HPF, Sirt3 $3^{-1}: 15.2 \pm 1.8$ vs. WT: $5.6 \pm 3.2$; casts/HPF, Sirt3 ${ }^{-}=10.9 \pm 3.2$ vs WT: $1.1 \pm 0.9$ ). Notably, while $87 \%$ of WT cisplatin-treated mice survived the 30 days that the experiment lasted, all Sirt3-deficient mice died within 7 days after cisplatin treatment (Figure 4D).

In order to exclude the possibility that the detrimental effect of SIRT3 deficiency on AKI was confined to the cisplatin model, we studied the impact of SIRT3 deficiency on the survival of animals with rhabdomyolysis-induced AKI caused by intramuscular injection of glycerol. The lack of SIRT3 led to the death of glycerol-treated mice. While all WT mice were alive, only $29 \%$ of Sirt3 $3^{-1-}$ littermates survived at 30 days $(P<0.01)$ (Supplemental Figure 5$)$.

Death of Sirt3-- AKI mice is not prevented by AICAR and $A L C A R$. To further document whether AICAR and ALCAR indeed protected against cisplatin-induced AKI by targeting SIRT3, we evaluated the effect of both compounds on the survival of $\mathrm{Sirt3}^{-1-}$ cisplatin-treated mice. Neither AICAR nor ALCAR was able to prolong the survival of Sirt $3^{--}$mice, which may suggest that SIRT3 is not dispensable for the protective effect of those molecules in AKI (Figure 4, E and F).

SIRT3 prevents cisplatin-induced mitochondrial dysfunction in cultured tubular cells. The functional role of SIRT3 in mitochondrial 

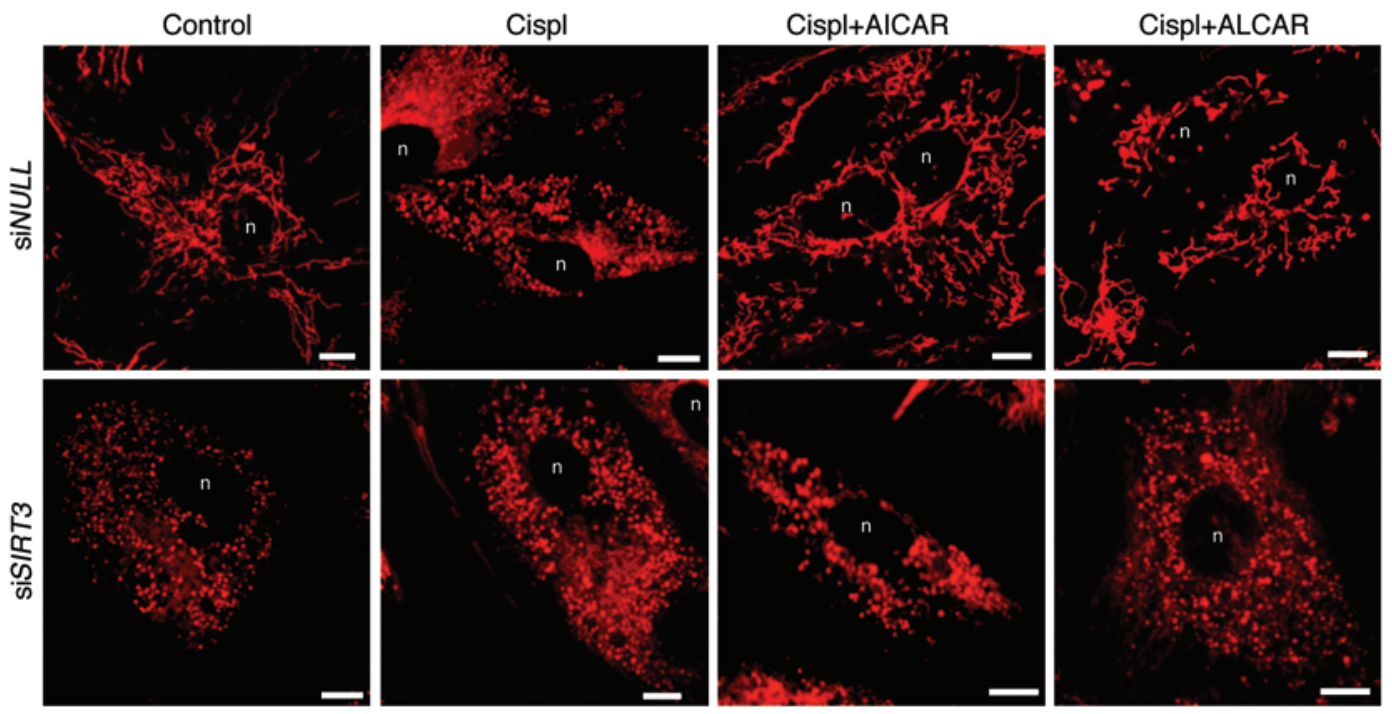

Figure 5. Role of SIRT3 in mitochondrial fragmentation in RPTECs exposed to cisplatin. Representative images showing mitochondrial morphology (visualized by staining with MitoTracker Deep Red) of RPTECs transfected with irrelevant siRNA (siNULL) or with SIRT3 siRNA (siSIRT3) and then exposed to medium or cisplatin ( $5 \mu \mathrm{M}$ for 24 hours) in the presence or absence of AICAR ( $2 \mathrm{mM}$ ) or ALCAR (1 mM). Scale bars: $10 \mu \mathrm{m}$. $\mathrm{n}, \mathrm{nucleus}$.

dynamics during AKI was further defined in an in vitro model of human renal proximal tubular epithelial cells (RPTECs) damaged by cisplatin. Consistent with the in vivo data, cisplatin exposure induced downregulation of SIRT3 mRNA $(0.6 \pm 0.08$-fold reduction in cisplatin-treated cells compared with control [1 \pm 0.05$]$, $P<0.05)$ accompanied by massive mitochondrial fragmentation (Figure 5). Treatments with AICAR and ALCAR restored SIRT3 mRNA $(1.6 \pm 0.2$ fold increase in cisplatin+AICAR-treated cells and $1.3 \pm 0.1$-fold increase in cisplatin+ALCAR-treated cells compared with control, with $P<0.001$ vs. cisplatin treatment alone) and mitochondrial network (Figure 5). That the two agents acted through SIRT3 is also supported by evidence that in RPTECs silenced with siRNA specific for SIRT3, both AICAR and ALCAR failed to rescue mitochondrial damage induced by cisplatin (Figure 5). Lipofectamine-mediated SIRT3 plasmid delivery in RPTECs yielded robust SIRT3 mRNA and protein expression (Supplemental Figure 6A). Concomitant with cisplatin-induced disruption of the mitochondrial network in RPTECs (Figure 6A) was the DRP1 recruitment in the organelles (Figure 6B) and the increase in the expression of the DRP1 receptor mitochondrial fission factor (MFF) (25) (Figure 6C). In SIRT3-transfected cells $(90 \% \pm 2.7 \%)$ exposed to cisplatin, mitochondrial integrity was preserved and organelle fragmentation was counteracted (Figure 6A) through the prevention of DRP1 recruitment (Figure 6B) and the inhibition of MFF upregulation in mitochondria (Figure 6C). SIRT3-dependent inhibition of the fission machinery was accompanied by increased expression of the fusion-promoting protein optic atrophy 1 (OPA1) (26), which was depressed by cisplatin (Figure 6, D and E, and Supplemental Figure 6B). Since OPA1 also positively regulates mitochondrial membrane potential $\left(\Delta \psi_{\mathrm{m}}\right)$ (27), the driving force for energy production, we used JC-1, a fluorescent dye highly sensitive to any small changes in $\Delta \psi_{\mathrm{m}}$ through a shift in the emission spectrum from green to red, reflecting cytoplasmic versus mitochondrial distribution. Cisplatin induced massive mitochondrial depolarization, as evidenced by diffuse cytoplasmic JC-1 green staining (Figure 7A). SIRT3 overexpression in cisplatin-damaged RPTECs restored mitochondrial membrane potential, allowing JC-1 uptake by mitochondria (red staining, Figure 7, A and B). Increased SIRT3 expression also conferred protection against mitophagy by reducing the cisplatininduced exuberant mitochondrial expression of PINK1 (Figure 7C) involved in the elimination of depolarized organelles (28).

\section{Discussion}

Here we found that in experimental AKI of mice, (a) renal mitochondrial SIRT3 was reduced; (b) Sirt3-deficient mice were more severely diseased and died; (c) pharmacological manipulations that increased SIRT3 protected WT but not Sirt3-deficient mice; and (d) the mechanism of SIRT3 renoprotection depended on its capacity to preserve mitochondria integrity, thereby limiting organelle fission and membrane depolarization.

Having established a strong association between renal insufficiency and reduced SIRT3 gene expression and protein synthesis, we first attempted to pharmacologically manipulate the system to clarify whether restoring mitochondrial SIRT3 could be protective. We first sought to activate AMPK, a critical regulator of mitochondria biogenesis in response to energy deprivation, to presumably induce SIRT3. In AKI mice given AICAR, renal expression of the rate-limiting enzyme NAMPT and SIRT3 normalized. The most surprising finding of our study is the electron microscopy evidence of SIRT3 downregulation in the mitochondria of proximal tubules of cisplatin-treated mice, which was totally rescued by AICAR. Findings of less tubular injury and protection from decline in renal function provide evidence that tubular damage in AKI can be a direct casualty of SIRT3 downregulation. Activating AMPK by AICAR increases NAMPT expression and mitochondrial protein content in skeletal muscle cells (20). On the other hand, NAMPT has a causative role in the regulation of SIRT3 gene transcription, based on previous data of ours showing that NAMPT gene silencing with specific siRNA prevented the decrease of SIRT3 mRNA 
A
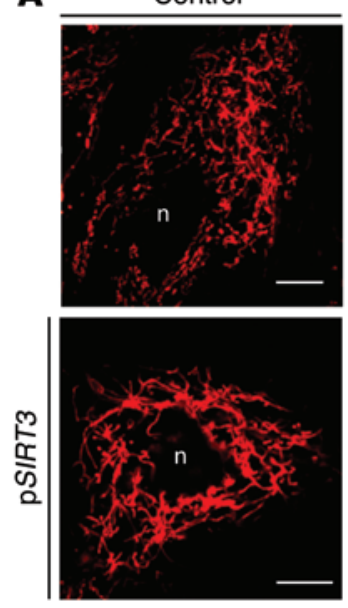

D
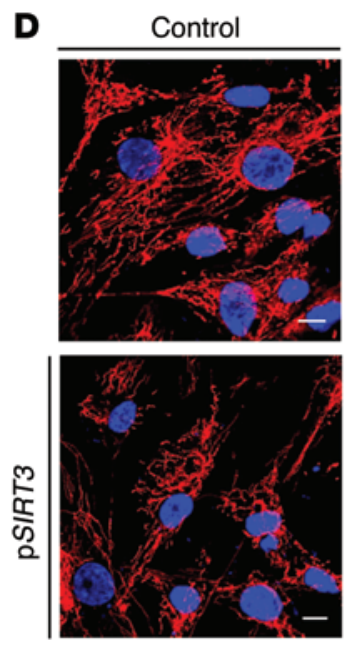

Cispl
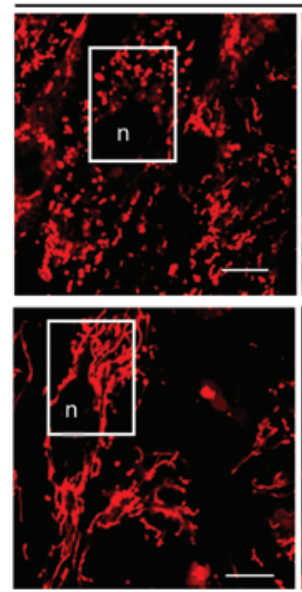

Cispl
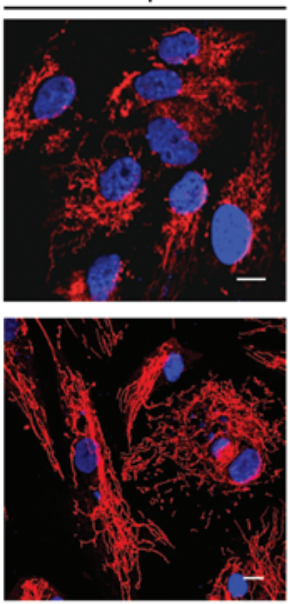

B

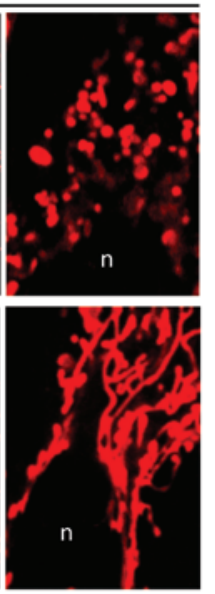



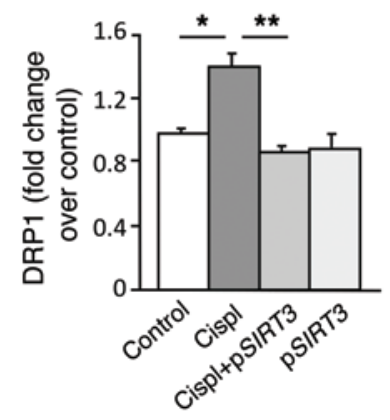

E

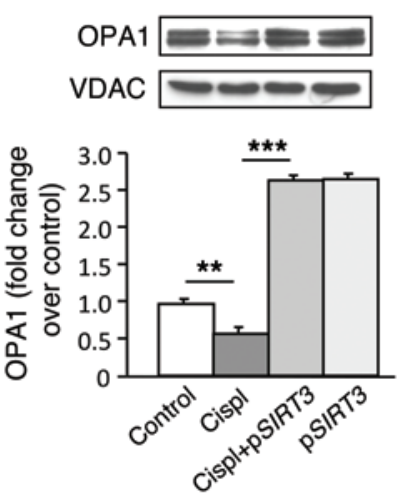

C
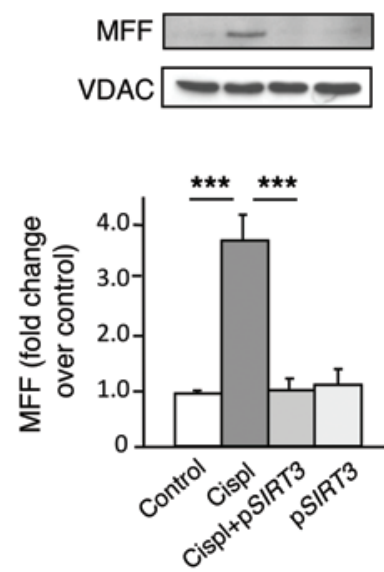

Figure 6. SIRT3 counteracts cisplatin-induced mitochondrial dynamic perturbation in injured tubular cells in culture. (A) Representative images showing a mitochondrial network of RPTECs untransfected (top) or transfected with SIRT3 plasmid (pSIRT3, bottom) and exposed to medium or cisplatin (5 $\mu$ M for 24 hours). Insets display fragmented (top) and elongated mitochondria (bottom) visualized by staining with MitoTracker Deep Red. Scale bars: $10 \mu \mathrm{m}$. (B and C) Representative Western blots and densitometric analysis of DRP1 and MFF protein expression in mitochondria isolated from control and cisplatin-treated RPTECs overexpressing or not $P$ SIRT3. VDAC protein expression was used as a sample loading control on the same membrane after stripping. ${ }^{*} P<0.05$, ${ }^{*} P<0.01$, and ${ }^{* *} P<0.001$, ANOVA corrected with Bonferroni coefficient. Values are mean \pm SEM $(n=3$ experiments). (D) Representative images of OPA1 immunofluorescence staining in control and cisplatin-injured RPTECs untransfected (top) or transfected with pSIRT3 (bottom). Nuclei were counterstained with DAPI (blue). Scale bars: $10 \mu \mathrm{m}$. (E) Representative Western blot and densitometric analysis of OPA1 protein expression in mitochondria isolated from control or cisplatin-treated RPTECs overexpressing or not $p$ SIRT3. ${ }^{* *} P<0.01$, and ${ }^{* * *} P<0.001$, ANOVA corrected with Bonferroni coefficient. Values are mean \pm SEM ( $n=3$ experiments). VDAC blots reported in B, C, and $\mathbf{E}$ derive from the same gel.

induced by angiotensin II in cultured proximal tubular cells (17). We then tested whether AMPK activation in mice with cisplatininduced AKI had an impact on Pgcla expression. The rationale for looking at Pgc1a gene expression rested on the finding that in addition to NAMPT, activating AMPK controls PGC-1 $\alpha(22,23)$, a crucial modulator of mitochondrial function, which induces SIRT3 gene expression by binding to estrogen-related receptor elements mapped in the promoter region (14). Here we found that mice with cisplatin-induced AKI showed a marked decrease in the renal expression of Pgcla, which was robustly enhanced by AICAR treatment. Consistent with this finding, in a model of sepsisassociated AKI, PGC-1 $\alpha$ was suppressed in proportion to the severity of renal damage and restored during the recovery phase (29), and proximal tubule-specific PGC-1 $\alpha$ deficiency exacerbated renal dysfunction during endotoxemia (29). Concomitant with the upregulation of proximal tubular SIRT3 expression induced by AICAR, we found that mitochondrial protein acetylation was normalized, providing-evidence of the capacity of the AMPK agonist to restore SIRT3 deacetylase activity. Most of those proteins exert a well-known protective effect against oxidative stress and contribute to mitochondrial energy homeostasis, two crucial events in the pathophysiology of AKI. While free oxygen radical generation in cisplatin-treated mice disrupted the equilibrium between mitochondrial fusion and fission (6), SIRT3 promoted mitochondrial biogenesis via PGC-1 $\alpha$-mediated inhibition of cellular ROS (14). The morphologic appearance of mitochondria rests on a balance between fission and fusion, with fragmentation resulting in more fission or less fusion. Thus, the stark change in the 
A
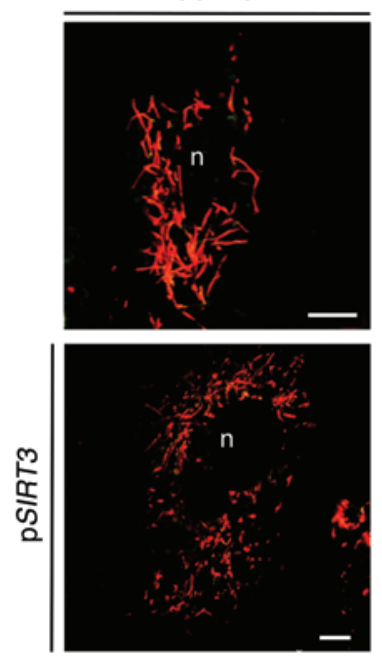

B

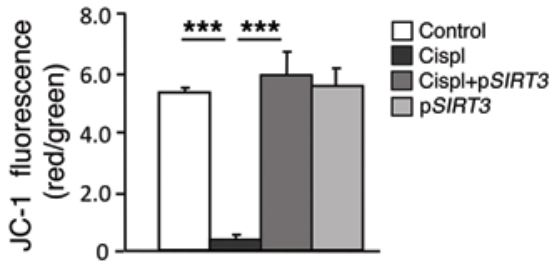

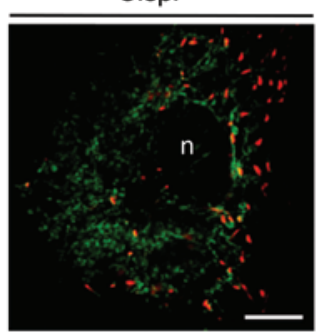

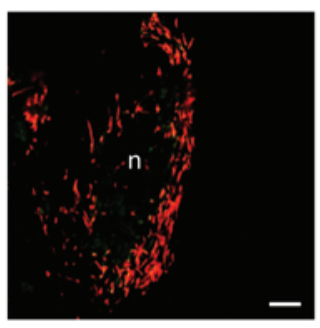

C

Control
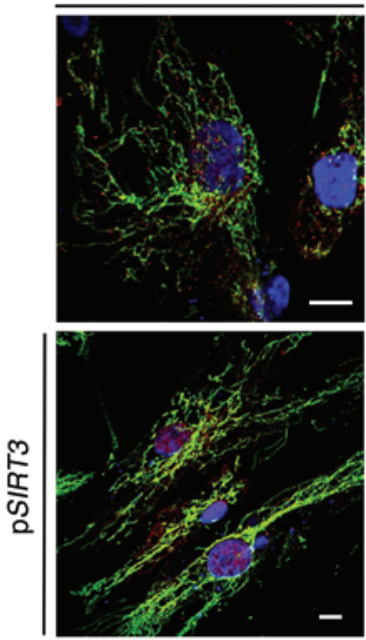

PINK1 hMT DAPI
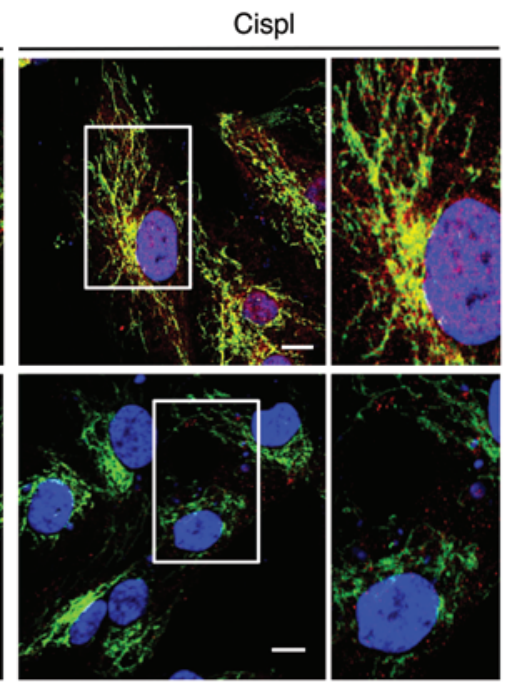

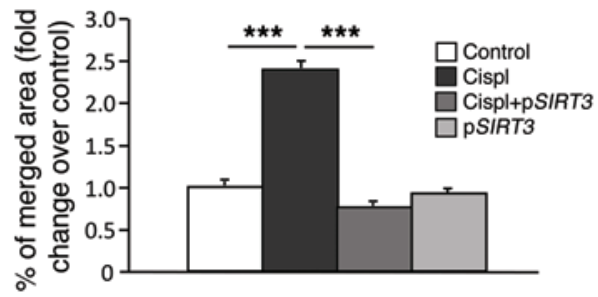

Figure 7. SIRT3 preserves mitochondrial integrity by limiting mitochondrial membrane depolarization and PINK1-related mitophagy in response to cisplatin. (A) Mitochondrial membrane potential visualized by JC-1, a dye sensitive to mitochondrial $\Delta \psi_{\mathrm{m}}$ changes. Depending on JC- 1 distribution, the emission spectrum of the dye shifts from green (cytoplasmic) to red (mitochondrial) in control or cisplatin-treated RPTECs untransfected (top) or transfected with pSIRT3 (bottom). (B) Quantification of JC-1 fluorescence intensity (red/green florescent area). Scale bars: $10 \mu \mathrm{m}$. ${ }^{* * *} P<0.001$ using ANOVA corrected with Bonferroni coefficient. Values are mean \pm SEM ( $n=3$ experiments). (C) Representative images of PINK1 distribution in control or cisplatin-treated RPTECs untransfected (top) or transfected with PSIRT3 (bottom) and costained with anti-human mitochondria antibody (hMT). Insets display the mitochondrial localization of PINK1 in enlarged images. Nuclei were counterstained with DAPI (blue). Scale bars: $10 \mu$ m. Quantification of merged fluorescence (yellow) of PINK1- (red) and hMT-positive mitochondrial staining (green) expressed as fold changes over control. ${ }^{* * *} P<0.001$, ANOVA corrected with Bonferroni coefficient. Values are mean $\pm \operatorname{SEM}(n=3$ experiments).

morphology and distribution of tubular mitochondria associated with an AICAR-mediated reduction in levels of the fission protein DRP1 highlights the clear beneficial role of AMPK activators in mitochondrial dynamics in the setting of AKI. Given the growing recognition of AMPK in diabetic and nondiabetic chronic kidney disease, these data underscore the therapeutic benefit of AMPK activation in these diseases (30). On the other hand, the biological relevance of the finding that DRP1 caused mitochondrial fragmentation, leading to tubular cell apoptosis and renal damage, rests on data showing that in both tubular cell cultures and rodent models of AKI, suppression of this protein through gene silencing and/or pharmacological inhibition limited mitochondrial fission, thus preserving their integrity (9).

To gain further insight into the possibility that oxidative stress-mediated cell death was indeed dependent on SIRT3 downregulation and could theoretically be prevented by pharmacological modulation of oxidation, we used the antioxidant agent ALCAR, which surprisingly did upregulate NAMPT and SIRT3, and limited renal dysfunction. The renoprotective effect of ALCAR in cisplatin-treated mice is reminiscent of that of resveratrol, a molecule with robust antioxidant properties that also protects cardiomyocytes from oxidative stress-induced death by modulating the SIRT pathway (16). In our experimental setting, ALCAR increased the number of mitochondria and limited their morphological alterations, an effect that can possibly be attributed to the activation of the transcription factor PGC- $1 \alpha$, which is instrumental in mitochondrial biogenesis.

Besides their implications for AKI pathophysiology, the present findings in mice have an obvious clinical correlate, recapitulated by animal survival data. We were surprised to find that all Sirt3-deficient mice, unlike their WT littermates, died within 7 days after cisplatin treatment, indicating that the SIRT3 pathway is a non-redundant player in tubular injury in AKI. The effect of SIRT3 deficiency on animal survival was not confined to cisplatininduced AKI but could be confirmed in another model. That SIRT3 was actually not dispensable in preventing mitochondrial damage and allowed for its repair in AKI is further documented by present findings in Sirt3-deficient animals. The latter were fully resistant to the therapeutic effect of both AICAR and ALCAR, which had, in stark contrast, been consistently beneficial in SIRT3-competent mice. Furthermore, the possibility that AICAR and ALCAR exert their action via SIRT3 is additionally supported by findings 


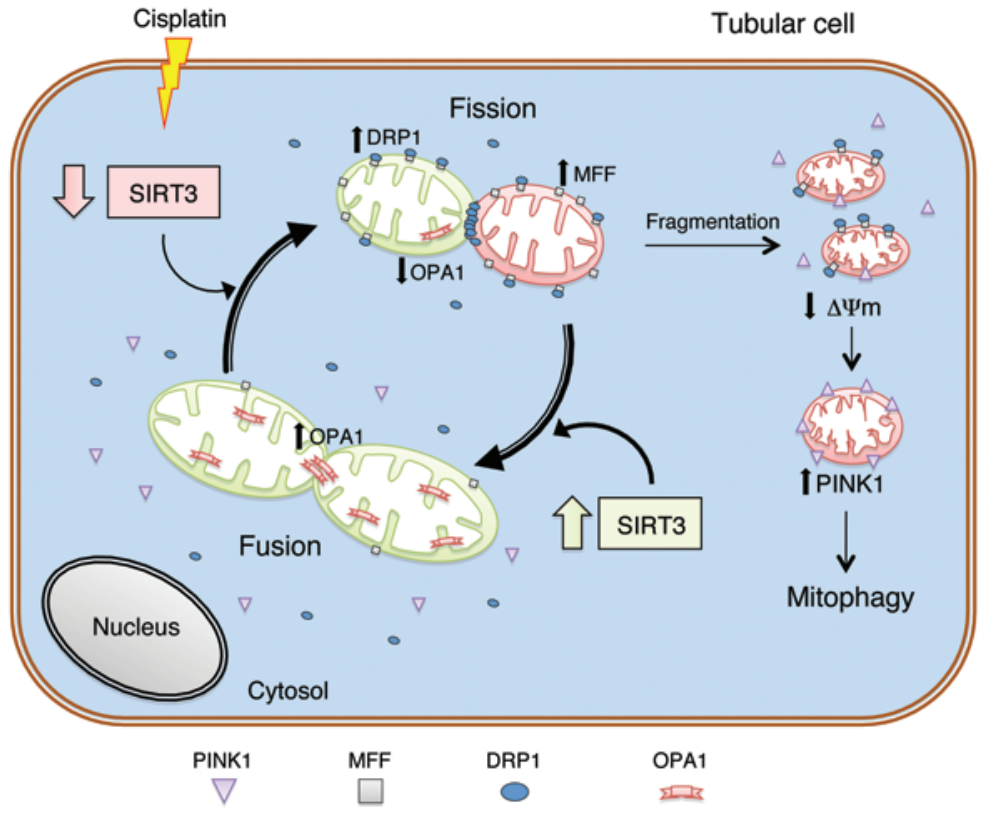

Figure 8. Schematic illustration of SIRT3's protective role in mitochondrial functional integrity in AKI. Cisplatin-induced SIRT3 downregulation causes increased expression of MFF and recruitment of DRP1 on the mitochondrial outer membrane, accompanied by a decrease in OPA1, thus carrying mitochondria dynamics toward fission and fragmentation. Following fission events, sustained mitochondrial depolarization triggers accumulation of PINK1 in the organelles, leading to activation of the mitophagic machinery. Upregulation of SIRT3 results in mitochondrial fusion, limiting segregation and depolarization, which represent the prerequisites for mitophagic processes. SIRT3 overexpression counterbalances the mitochondrial dysfunction through the reduction of mitochondrial MFF/DRP1 expression balanced by upregulation of OPA1, preservation of $\Delta \psi_{\mathrm{m}}$, and inhibition of PINK1. that in RPTECs silenced for SIRT3, both compounds failed to rescue mitochondrial fragmentation induced by cisplatin. Taken together, the above evidence that the two drugs have the capacity to upregulate SIRT3 via different pathways and the present unanticipated findings in Sirt3-deficient animals and RPTECs exposed to both compounds indicate that SIRT3 is possibly a key, albeit not the sole, mechanism by which AICAR and ALCAR protect the kidney and other organs (23) from oxidative damage in many disease conditions.

The biological relevance of SIRT3 in AKI is also highlighted by in vitro findings showing its role in the maintenance of mitochondrial homeostasis. Mitochondria fission is catalyzed by DRP1, which is recruited by MFF anchored to the outer membrane of the organelle in order to form spirals to sever both mitochondrial membranes $(7,8,25)$. In the face of high SIRT3 expression, mitochondrial fragmentation induced by cisplatin was limited through the normalization of DRP1 and MFF protein levels (see schematic, Figure 8). Another element tipping the balance toward fusion is the SIRT3-induced upregulation of OPA1, which mediates the fusion of the inner membrane of the organelle $(8,26,27)$. Alternatively, one can consider the possibility that overexpressing SIRT3 prevents a cisplatin-induced decrease in OPA1. SIRT3induced restoration of OPA1 counteracts mitochondrial membrane depolarization to the extent that $\Delta \psi_{\mathrm{m}}$ normalized, accomplishing an essential condition for the production of ATP through respiration (31). An additional consequence of cisplatin-induced mitochondrial fragmentation and depolarization is the translocation on mitochondrial membrane of PINK1, a key trigger of mitophagy $(28,32)$, which was completely normalized by SIRT3 overexpression, suggesting SIRT3 as one of the crucial regulators of mitochondrial functional integrity in cisplatin-induced AKI.

The obvious translational consequence of these in vivo and in vitro studies will be to look for potential SIRT3-activating compounds, which the current pharmacopeia is remarkably lacking. Should one of those molecules become available in future for human use, individuals with many chronic conditions still lacking effective treatment, including metabolic syndrome, premature aging, and cancer, could benefit. Our present findings add AKI to this list. The search for specific SIRT3 activators may offer hope to patients who still die of a condition that is often preventable and claims 1.7 million lives per year globally.

\section{Methods}

Experimental design. Two-month-old C57BL/6J, C57BL6 × 129 (Charles River Italia Srl), and Sirt3 ${ }^{--}$female mice (provided by Frederick Alt, Harvard Medical School, Boston, Massachusetts, USA) were used. Sirt3 $3^{-/}$mice were generated in a mixed genetic background as previously described (11). Animals were housed in a constant-temperature room $\left(22-24^{\circ} \mathrm{C}\right)$ with a 12 -hour dark/12-hour light cycle and fed a standard diet. AKI was induced by subcutaneous injection of cis-diamminedichloroplatinum (cisplatin $17.4 \mathrm{mg} / \mathrm{kg}$; Ebewe Italia Srl) or by an intramuscular injection with hypertonic glycerol $(8 \mathrm{ml} / \mathrm{kg}$ body wt of a $50 \%$ glycerol solution; Sigma-Aldrich) into the inferior hind limbs. To investigate the effects of AICAR (Toronto Research Chemicals Inc.) and ALCAR (Sigma-Tau Pharmaceuticals Inc.) in the cisplatin-induced AKI model, C57BL/6J and Sirt3 ${ }^{--}$mice receiving cisplatin were i.p. injected 24 hours later with AICAR (500 mg/kg) (33) or ALCAR (200 mg/kg) (34) for 3 days. The mice were then sacrificed 4 days after cisplatin treatment, and kidney samples were used for histology and immunohistochemistry evaluations. Normal mice served as the control. To assess survival, an additional group of C57BL6 $\times 129$ cisplatin-treated mice were compared with cisplatin-treated Sirt3 ${ }^{-/}$mice. The effect of AICAR or ALCAR on survival of Sirt3 ${ }^{-/-}$mice with cisplatin-induced AKI was also studied.

Renal function was assessed as BUN using the Reflotron test (Roche Diagnostics) and as serum creatinine using the Cobas Mira Plus autoanalyzer (Roche Diagnostics) according to the manufacturer's instructions.

Estimation of mitochondrial numerical density and mean mitochondrial volume. Glutaraldehyde-fixed fragments of cortical kidney tissue were washed repeatedly in cacodylate buffer, postfixed in $1 \% \mathrm{OsO}_{4}$, dehydrated through ascending grades of alcohol, and embedded in Epon resin. Ultrathin sections were stained with uranyl acetate for examination using a Philips Morgagni transmission electron micro- 
scope (TEM). Numerical density of mitochondria $\left(N_{V}, n / \mu \mathrm{m}^{3}\right)$ was estimated using morphometric analysis according to Weibel (35), using an orthogonal grid digitally superimposed onto digitized electron microscope pictures of proximal tubules at $\times 7,100$. Briefly, the mitochondrial profile area density $\left(N_{A}\right)$ was estimated using the ratio between the number of mitochondria and the proximal tubular area in the image calculated on the basis of grid points. Mitochondrial volume density $\left(V_{V}\right)$ was determined using the ratio of grid points falling over mitochondria divided by the total number of points of the grid container in the proximal tubule section. $N_{V}$ was then estimated for each animal using the following formula (35): $N_{V}=(1 / \beta)\left(N_{A}^{3 / 2} / V_{V}^{1 / 2}\right)$, where $\beta$ is the shape coefficient for ellipsoidal mitochondria, calculated using the ratio of the harmonic mean of major and minor axes of mitochondrial sections measured on digital images. The mean mitochondrial volume was calculated for each animal as the ratio of $V_{V}$ to $N_{V}$.

Oxidative damage. To determine protein nitration of tyrosine residues, paraffin kidney sections were fixed with methanol and incubated with $30 \% \mathrm{H}_{2} \mathrm{O}_{2}$ for 30 minutes and then with $0.3 \%$ Triton $\mathrm{X}-100$ in PBS for 15 minutes. Sections were incubated with a rabbit polyclonal anti-nitrotyrosine primary antibody (1:2,000, Upstate Biotechnology), followed by biotinylated goat anti-rabbit IgG secondary antibody (1:200, Vector Laboratories). Signal was developed using the Vectastain $\mathrm{ABC}$ kit and DAB reagents (Vector Laboratories). Negative controls were performed omitting the primary antibody. At least 30-40 non-overlapping sequential fields were analyzed. Each section was scored for intensity (0: absent, 1: faint, 2: moderate, 3: intense).

Renal histology. Kidney samples were fixed in Duboscq-Brazil, and paraffin sections were stained with periodic acid-Schiff (PAS). Luminal hyaline casts and tubular necrosis (denudation of the tubular basement membrane) were assessed in non-overlapping fields (up to 28 for each section) using a $\times 40$ objective (HPF; Primo Start, Zeiss). The number of casts and tubular profiles showing necrosis was analyzed twice in a single-blind fashion.

Immunofluorescence analysis in renal tissue. For SIRT3 staining in renal tissue, $3-\mu \mathrm{m}$ periodate-lysine-paraformaldehyde-fixed (periodate-lysine-PFA-fixed) cryosections were air dried and washed with PBS. After blocking nonspecific sites with $1 \%$ BSA, slides were incubated with goat anti-mouse SIRT3 (1:25, Santa Cruz Biotechnology Inc.) and rabbit anti-mouse VDAC (1:50, Sigma-Aldrich) antibodies, followed by rabbit anti-goat FITC-conjugated (1:80, Jackson ImmunoResearch Laboratories) or donkey anti-mouse Cy3-conjugated secondary antibodies (1:80, Jackson ImmunoResearch Laboratories), respectively. Nuclei were stained with DAPI (Sigma-Aldrich), and the renal structure was labeled with rhodamine-labeled lens culinaris agglutinin (1:400; Vector Laboratories). SIRT3 quantification was examined in at least 25 fields for each animal by using the appropriate software (Axio Vision, Apotome Axio Imager Z2, Zeiss) and expressed as the SIRT3-positive area $\left(\mu \mathrm{m}^{2}\right)$ in each field.

Immunoelectron microscopy. Fragments of periodate-lysine-PFAfixed renal cortex were infiltrated with $2.3 \mathrm{M}$ sucrose for at least 1 hour, sectioned, and transferred to nickel grids coated with Formvar (Electron Microscopy Sciences). After blocking with 1\% BSA for 15 minutes, sections were incubated with goat anti-mouse SIRT3 antibody (1:400, Santa Cruz Biotechnology Inc.), followed by $12 \mathrm{~nm}$ gold-conjugated donkey anti-goat IgG secondary antibody (1:50, Jackson ImmunoResearch Laboratories), then stained with $2 \%$ aqueous uranyl acetate and embedded in methylcellulose before being examined with TEM.
Cell culture and incubation. Human RPTECs were purchased from Lonza and grown in renal epithelial cell basal medium (REBM) supplemented with REGM SingleQuots (Lonza). To assess SIRT3 mRNA expression, RPTECs were exposed to $5 \mu \mathrm{M}$ cisplatin for 24 hours in the presence or absence of AICAR $(2 \mathrm{mM})$ or ALCAR $(1 \mathrm{mM})$ added 1 hour before and during cisplatin incubation.

SIRT3 silencing. RPTECs were transfected with Silencer Select predesigned siRNA human SIRT3 (s23768, Life Technologies) (100 pmol) or with control nontarget siRNA (Ambion, Silencer Select Negative Control \#2siRNA) using Lipofectamine 2000 reagent (Invitrogen) according to the manufacturer's protocol. Forty-eight hours after transfection, cells were incubated with $5 \mathrm{mM}$ cisplatin for 24 hours in the presence or absence of AICAR ( $2 \mathrm{mM})$ or ALCAR $(1 \mathrm{mM})$ added 1 hour before and during cisplatin incubation for MitoTracker Red (Molecular Probes, Invitrogen) assessment. SIRT3 silencing resulted in $70 \%$ inhibition of SIRT3 mRNA expression (siNULL: $0.93 \pm 0.17$, vs. SiSIRT3: $0.29 \pm 0.05$ arbitrary units, $P<0.01$ ).

SIRT3 overexpression. Cells seeded on $35-\mathrm{mm}^{2}$ tissue culture plates at $70 \%$ confluence were transfected with plasmid DNA (GFP-tagged pCMV-hSIRT3, Origene Technologies) using Lipofectamine 2000 (Invitrogen, Life Technologies) as described in the manufacturer's instructions. Briefly, cells were incubated with $5 \mu$ Lipofectamine 2000 reagent and $2.5 \mu \mathrm{g}$ plasmid DNA mixed in $2 \mathrm{ml}$ medium for 6 hours followed by fresh medium for 24 hours. Then, the RPTECs were exposed to medium with or without cisplatin ( $5 \mu \mathrm{M}$; Ebewe Italia Srl) for 24 hours.

Immunofluorescence analysis in cultured cells. Cells were fixed in 2\% PFA (Electron Microscopy Science) and 4\% sucrose (SigmaAldrich), followed by permeabilization with $0.3 \%$ Triton $\mathrm{X}-100$ (Sigma-Aldrich). Nonspecific binding sites were blocked with $2 \%$ FBS, $2 \%$ BSA, and $0.2 \%$ bovine gelatin. Cells were incubated with a mouse monoclonal anti-OPA1 antibody (1:50, BD Biosciences) or with a rabbit polyclonal anti-PINK1 antibody (1:50, Santa Cruz Biotechnology Inc.), followed by incubation with a donkey anti-mouse or goat anti-rabbit Cy3-conjugated secondary antibody (1:80, Jackson ImmunoResearch Laboratories), respectively. For colocalization studies, cells were then incubated with a mouse monoclonal antihuman mitochondria antibody (hMT, 1:50, Millipore) and a goat antimouse FITC-conjugated secondary antibody (1:80, Jackson ImmunoResearch Laboratories). Nuclei were counterstained with DAPI (Sigma-Aldrich). Negative controls were obtained by omitting primary antibodies. Samples were examined using a confocal inverted laser microscope (LSM 510 Meta, Zeiss). The quantification of the area corresponding to OPA1 staining was performed in 15 random fields per sample, expressed as pixel ${ }^{2}$ (ImageJ 1.40g software; http:// imagej.nih.gov/ij/), and normalized for the number of DAPI-positive cell nuclei. The quantification of the merged fluorescence (yellow area) of PINK1 expression (red) and hMT-positive mitochondria (green) was performed in 15 random fields per sample.

Mitochondrial morphology and membrane potential detection. Mitochondria were labeled by incubating living cells with the fluorescent probe MitoTracker Red (250 nM for 30 minutes, Molecular Probes, Invitrogen). Mitochondrial membrane potential was evaluated by exposing cells to JC-1 (5 $\mu \mathrm{M}$ for 30 minutes, Molecular Probes, Invitrogen), a dye exhibiting potential-dependent accumulation in mitochondria indicated by a fluorescence shift from green (cytoplasm) to red (mitochondria). At the end of incubations, cells were examined by confocal inverted laser microscopy; quantification of JC-1 red and 
green areas (pixel ${ }^{2}$ ) was performed in 15 random fields per sample; and mitochondrial membrane potential was expressed as the ratio of red to green fluorescence areas.

Real-time PCR. Mouse renal tissue and human RPTECs were harvested in TRIzol reagent (Invitrogen, Life Technologies), and total RNA was extracted according to the manufacturer's instructions. Contaminating genomic DNA was removed by RNase-free DNase (Promega) for 1 hour at $37^{\circ} \mathrm{C}$. The first-strand cDNA $(2 \mu \mathrm{g})$ was produced using a SuperScript VILO cDNA Synthesis Kit (Life Technologies) according to the manufacturer's procedure. No enzyme was added for reverse transcriptase-negative controls ( $\left.\mathrm{RT}^{-}{ }^{-}\right)$.

To amplify cDNA of mouse Nampt, Pgcla, and Sirt3, we used TaqMan Universal PCR Master Mix (Applied Biosystems) and inventoried TaqMan assays of the Nampt gene (FAM/MBG probe Mm01293560m1), the Pgc1a gene (FAM/MBG probe Mm00447181m1), the Sirt3 gene (FAM/MBG probe Mm01275638m1), and a mouse $\alpha$-actin endogenous control (VIC/MGB probe) according to the manufacturer's instructions. PCR was performed on the Viia7 Real-Time PCR System (Applied Biosystems). The amplification profile consisted of 2 minutes at $50^{\circ} \mathrm{C}$ and 10 minutes at $95^{\circ} \mathrm{C}$; the samples were cycled 40 times at $95^{\circ} \mathrm{C}$ for 15 seconds and $60^{\circ} \mathrm{C}$ for 60 seconds. Data were analyzed using the $2^{-\triangle \Delta \mathrm{CT}}$ method and presented as fold changes relative to mouse wild type (control).

cDNA of human SIRT3 and GAPDH was amplified using inventoried TaqMan assays (FAM/MBG probe Hs_00202030m1 and $4333764 \mathrm{~F}$, respectively) according to the manufacturer's instructions.

Protein extraction from renal tissue and mitochondria. Renal tissues were homogenized in buffer (50 mM HEPES pH 7.4, $150 \mathrm{mM}$ $\mathrm{NaCl}, 1 \mathrm{mM}$ EDTA, $1 \mathrm{mM}$ EGTA, $0.5 \mathrm{mM} \mathrm{NaVO}_{4}, 10 \mathrm{mM}$ Na fluoride, $1 \mathrm{mM} \beta$-glycerophosphate, $20 \mathrm{mM} \mathrm{H}_{3} \mathrm{NaO}_{7} \mathrm{P}_{2}, 10 \%$ glycerol, $1 \%$ Triton $\mathrm{X}-100$, supplemented with protease inhibitor cocktail, Sigma-Aldrich) and centrifuged $15,000 \mathrm{~g}$ for 15 minutes at $4^{\circ} \mathrm{C}$ to remove detergentinsoluble material. Mitochondria were isolated from mouse renal tissue or human RPTECs using the Qproteome Mitochondria Isolation Kit (QIAGEN) according to the manufacturer's protocol. Isolated mitochondria were solubilized in an appropriate buffer $(150 \mathrm{mM} \mathrm{NaCl}$, $50 \mathrm{mM}$ Tris- $\mathrm{HCl} \mathrm{pH} \mathrm{7.4,} 10 \mathrm{mM}$ nicotinamide, $500 \mathrm{nM}$ trichostatin A, $1 \% n$-dodecyl- $\beta$-maltoside). Mitochondrial and total protein concentration was determined using a DC assay (Bio-Rad).

Western blot analysis. Equal amounts of proteins were separated on SDS-PAGE under reducing conditions and transferred to PVDF membranes (Bio-Rad). After blocking with 5\% BSA in TBS supplemented with $0.1 \%$ Tween-20, membranes were incubated with the following antibodies: rabbit anti-phospho-AMPK $\alpha$ (1:1,000, Cell Signaling Technology), anti-total AMPK $\alpha$ antibody (1:1,000, Cell Signaling Technology), rabbit anti-acetylated lysine antibody (1:1,000, Cell Signaling Technology), mouse anti-DRP1 antibody (1:2,000, BD Biosciences), rabbit anti-MFF (1:1,000, Origene Technologies), mouse anti-OPA1 antibody (1:1,000, BD Biosciences), and rabbit anti-VDAC antibody (1:1000, Cell Signaling Technology). VDAC was used as a sample loading control in isolated mitochondria after stripping the same membranes. The signal was visualized using the corresponding horseradish peroxidase-conjugated secondary antibodies (1:30,000; Sigma-Aldrich) and enhanced chemiluminescence-Western Blotting Detection Reagent (Pierce, Thermo Fisher Scientific Inc.). Bands were quantified by densitometry using Image $1.40 \mathrm{~g}$ software.

Statistics. Data are expressed as mean \pm SEM and in box-plot diagrams. Data analysis was performed using Prism software (GraphPad Software Inc.). Comparisons were made using ANOVA with the Bonferroni post hoc test or unpaired Student's $t$ test as appropriate. Statistical significance was defined as $P<0.05$. Survival curves were calculated according to the log-rank test.

Study approval. All procedures involving mice were performed in accordance with institutional guidelines that are in compliance with national (D.L. n.116, G.U., suppl. 40, February 18, 1992, circolare no. 8, G.U., July 14, 1994) and international laws and policies (EEC Council Directive 86/609, OJL, no. 358, December 1987; Guide for the Care and Use of Laboratory Animals, National Academy Press, 1996) and were approved by the Institutional Animal Care and Use Committees of Mario Negri Institute, Milan, Italy.

\section{Acknowledgments}

We are greatly indebted to Frederick Alt for providing Sirt3-knockout mice. We thank Paola Cassis for nitrotyrosine experiments and Manuela Passera for help in preparing the manuscript. This work was supported by a grant from fiRSt Srl, Rome, Italy.

Address correspondence to: Giuseppe Remuzzi, IRCCS - Istituto di Ricerche Farmacologiche "Mario Negri," Centro Anna Maria Astori, Science \& Technology Park Kilometro Rosso, Via Stezzano, 87, 24126 Bergamo, Italy. Phone: 39.035.42131; E-mail: giuseppe. remuzzi@marionegri.it.
1. Lewington AJ, Cerda J, Mehta RL. Raising awareness of acute kidney injury: a global perspective of a silent killer. Kidney Int. 2013;84(3):457-467.

2. Acute kidney injury: prevention, detection and management of acute kidney injury up to the point of renal replacement therapy. NICE Web site. https://www.nice.org.uk/guidance/cg169. Accessed December 11, 2014.

3. Pabla N, Dong Z. Cisplatin nephrotoxicity: mechanisms and renoprotective strategies. Kidney Int. 2008;73(9):994-1007.

4. Thadhani R, Pascual M, Bonventre JV. Acute renal failure. N Engl JMed. 1996;334(22):1448-1460.

5. Bonventre JV, Yang L. Cellular pathophysiology of ischemic acute kidney injury. JClin Invest. 2011;121(11):4210-4221.

6. Zhan M, Brooks C, Liu F, Sun L, Dong Z. Mito- chondrial dynamics: regulatory mechanisms and emerging role in renal pathophysiology. Kidney Int. 2013;83(4):568-581.

7. Palmer CS, Osellame LD, Stojanovski D, Ryan MT. The regulation of mitochondrial morphology: intricate mechanisms and dynamic machinery. Cell Signal. 2011;23(10):1534-1545.

8. Friedman JR, Nunnari J. Mitochondrial form and function. Nature. 2014;505(7483):335-343.

9. Brooks C, Wei Q, Cho SG, Dong Z. Regulation of mitochondrial dynamics in acute kidney injury in cell culture and rodent models. JClin Invest. 2009;119(5):1275-1285.

10. Baur JA, Ungvari Z, Minor RK, Le Couteur DG, de Cabo R. Are sirtuins viable targets for improving healthspan and lifespan? Nat Rev Drug Discov. 2012;11(6):443-461.
11. Lombard DB, et al. Mammalian Sir2 homolog SIRT3 regulates global mitochondrial lysine acetylation. Mol Cell Biol. 2007;27(24):8807-8814.

12. Ahn BH, et al. A role for the mitochondrial deacetylase Sirt3 in regulating energy homeostasis. Proc Natl Acad Sci US A. 2008;105(38):14447-14452.

13. Wu YT, Lee HC, Liao CC, Wei YH. Regulation of mitochondrial $\mathrm{F}(\mathrm{o}) \mathrm{F}(1)$ ATPase activity by Sirt3-catalyzed deacetylation and its deficiency in human cells harboring $4977 \mathrm{bp}$ deletion of mitochondrial DNA. Biochim Biophys Acta. 2013;1832(1):216-227.

14. Kong X, et al. Sirtuin 3, a new target of PGC-1alpha, plays an important role in the suppression of ROS and mitochondrial biogenesis. PLoS One. 2010;5(7):e11707. 
15. Bell EL, Guarente L. The SirT3 divining rod points to oxidative stress. Mol Cell. 2011;42(5):561-568.

16. Chen CJ, Fu YC, Yu W, Wang W. SIRT3 protects cardiomyocytes from oxidative stress-mediated cell death by activating NF- $\mathrm{\kappa B}$. Biochem Biophys Res Commun. 2013;430(2):798-803.

17. Benigni A, et al. Disruption of the Ang II type 1 receptor promotes longevity in mice. JClin Invest. 2009;119(3):524-530.

18. Morigi M, et al. Mesenchymal stem cells are renotropic, helping to repair the kidney and improve function in acute renal failure. J Am Soc Nephrol. 2004;15(7):1794-1804.

19. Imai S. Nicotinamide phosphoribosyltransferase (Nampt): a link between NAD biology, metabolism, and diseases. Curr Pharm Des. 2009;15(1):20-28.

20. Costford SR, et al. Skeletal muscle NAMPT is induced by exercise in humans. Am J Physiol Endocrinol Metab. 2010;298(1):E117-E126.

21. Fulco M, et al. Glucose restriction inhibits skeletal myoblast differentiation by activating SIRT1 through AMPK-mediated regulation of Nampt. Dev Cell. 2008;14(5):661-673.
22. Zong $\mathrm{H}$, et al. AMP kinase is required for mitochondrial biogenesis in skeletal muscle in response to chronic energy deprivation. Proc Natl Acad Sci U S A. 2002;99(25):15983-15987.

23. Canto $C$, et al. AMPK regulates energy expenditure by modulating $\mathrm{NAD}^{+}$metabolism and SIRT1 activity. Nature. 2009;458(7241):1056-1060.

24. Tufekci O, et al. Evaluation of the effect of acetyl L-carnitine on experimental cisplatin nephrotoxicity. Chemotherapy. 2009;55(6):451-459.

25. Otera $\mathrm{H}$, et al. Mff is an essential factor for mitochondrial recruitment of Drp1 during mitochondrial fission in mammalian cells. J Cell Biol. 2010;191(6):1141-1158.

26. Cipolat S, Martins de Brito O, Dal Zilio B, Scorrano $L$. OPA1 requires mitofusin 1 to promote mitochondrial fusion. Proc Natl Acad Sci U S A. 2004;101(45):15927-15932.

27. Samant SA, et al. SIRT3 deacetylates and activates OPA1 to regulate mitochondrial dynamics during stress. Mol Cell Biol. 2014;34(5):807-819.

28. Thomas RL, Gustafsson AB. Mitochondrial autophagy - an essential quality control mechanism for myocardial homeostasis. Circ J. 2013;77(10):2449-2454.
29. Tran M, et al. PGC-1 $\alpha$ promotes recovery after acute kidney injury during systemic inflammation in mice. JClin Invest. 2011;121(10):4003-4014.

30. Dugan LL, et al. AMPK dysregulation promotes diabetes-related reduction of superoxide and mitochondrial function. JClin Invest. 2013;123(11):4888-4899.

31. Hoppins $S$. The regulation of mitochondrial dynamics. Curr Opin Cell Biol. 2014;29:46-52.

32. Twig G, Shirihai OS. The interplay between mitochondrial dynamics and mitophagy. Antioxid Redox Signal. 2011;14(10):1939-1951.

33. Bai A, et al. AMPK agonist downregulates innate and adaptive immune responses in TNBSinduced murine acute and relapsing colitis. Biochem Pharmacol. 2010;80(11):1708-1717.

34. Xia Y, Li Q, Zhong W, Dong J, Wang Z, Wang C. L-carnitine ameliorated fatty liver in highcalorie diet/STZ-induced type 2 diabetic mice by improving mitochondrial function. Diabetol Metab Syndr. 2011;3:31.

35. Weibel ER. Practical methods for biological morphometry. In: Stereological Methods. Vol. 1. London, United Kingdom: Academic Press; 1979:40-116. 